\title{
A fluorescent amyloid sensor for quantitative super- resolution imaging of amyloid fibril assembly.
}

\author{
Amandeep Kaur, ${ }^{* 1,2}$ Liam D. Adair, ${ }^{3,4}$ Sarah R. Ball, ${ }^{1}$ Elizabeth J. New,,${ }^{3,4}$ Margaret Sunde ${ }^{1,2}$
}

${ }^{1}$ The University of Sydney, School of Medical Sciences, Faculty of Medicine and Health, NSW 2006, Australia

${ }^{2}$ The University of Sydney Nano Institute (Sydney Nano), The University of Sydney, NSW 2006, Australia

${ }^{3}$ The University of Sydney, School of Chemistry, NSW 2006, Australia

${ }^{4}$ Australian Research Council Centre of Excellence for Innovations in Peptide and Protein Science, The University of

Sydney, NSW 2006, Australia

\begin{abstract}
Many soluble proteins can self-assemble into macromolecular structures called amyloids, a subset of which are implicated in a range of neurodegenerative disorders. The nanoscale size and structural heterogeneity of prefibrillar and early aggregates, as well as mature amyloid fibrils, pose significant challenges for the quantification of amyloid species, identification of their cellular interaction partners and for elucidation of the molecular basis for cytotoxicity. We report a fluorescent amyloid sensor AmyBlink-1 and its application in super-resolution imaging of amyloid structures. AmyBlink-1 exhibits a 5 -fold increase in ratio of the green (thioflavin T) to red (Alexa Fluor 647) emission intensities upon interaction with amyloid fibrils. Using AmyBlink-1, we performed nanoscale imaging of four different types of amyloid fibrils, achieving a resolution of $\sim 30 \mathrm{~nm}$. AmyBlink-1 enables molecular-level visualization and subsequent quantification of morphological features, such as the length and skew of individual amyloid aggregates formed at different times along the amyloid assembly pathway.
\end{abstract}

Amyloids are characterized by an organized macromolecular architecture, composed of extended $\beta$-sheets, which is generated through the self-assembly of proteins. ${ }^{[1]}$ Pathological, artificial and functional amyloids share this hallmark cross $-\beta$ structure. Protein misfolding and aggregation into amyloid fibrils is a feature in many diseases including Alzheimer's disease (AD) and Parkinson's disease (PD), as well as type 2 diabetes and the prion-associated encephalopathies. ${ }^{[2]}$ The preponderance of readily detectable fibrillar structures has led to a heavy focus of therapeutic efforts towards these structures. However, recent studies suggest early stages of amyloid aggregation are responsible for the toxicity and pathology associated with neurodegeneration..$^{[3]}$ The heterogeneity of metastable early-stage aggregates and polymorphic nature of amyloid fibrils observed in later stages of disease have limited our understanding of the impact of fibril formation and deposition on cells and tissues. ${ }^{[3 a, 4]}$ Tools and technologies that enable nano-scale visualization and discrimination of amyloid structures are therefore highly sought after. ${ }^{[5]}$

Despite the advantages of standard optical microscopy, the application of the technique for nanoscale investigations is hampered by the resolution limit $(250-300 \mathrm{~nm})$ set by the diffraction of light. ${ }^{[6]}$ In the past decade, exciting new categories of super-resolution microscopy have been developed, particularly the single-molecule localization microscopy (SMLM) techniques such as dSTORM (direct stochastic optical reconstruction microscopy) and PALM (photoactivated localization microscopy) which require fluorophores to reversibly switch between bright and dark states. This creates transient spatial separation between the individual fluorophores, allowing for their localization at the nanometer-scale in a pointillist manner. ${ }^{[7]}$ In addition to delivering $\sim 10 \mathrm{~nm}$ lateral (xy) and $\sim 30 \mathrm{~nm}$ axial (z) resolution, dSTORM has been widely adopted by researchers due to the inexpensive equipment required and ease of optical alignment, unlike other techniques. ${ }^{[7]}$

Common approaches for nanoscale amyloid imaging involve labelling with genetically-encoded photoswitchable fluorescent proteins or antibodies tagged with photoswitchable dyes. ${ }^{[8]} \mathrm{A}$ limitation with these is that the distance between the fluorophore and protein of interest may be larger than the resolution regime $(10-20 \mathrm{~nm})$ of super-resolution microscopy, resulting in a significant linkage error. ${ }^{[9]}$ Small molecule fluorescent sensors do not suffer from this limitation, and offer the flexibility of achieving a ratiometric fluorescence response, nullifying artefacts arising from variations in sensor concentration, environment and instrumental inconsistencies. ${ }^{[10]}$ Amyloidophilic dyes such as Thioflavin T (ThT) specifically bind to hydrophobic grooves accessible on fibrils. ${ }^{[11]}$ Recently, detection of the transient binding of ThT to $A \beta$ has shown promise for nanoscale imaging of amyloids and SPAINT has provided aggregate hydrophobicity maps at this scale. ${ }^{[12]}$ However, ThT lacks photoswitching properties, as do most reported fluorescent amyloid sensors, ${ }^{[5,13]}$ and therefore cannot be used in dSTORM imaging experiments. 
Here, we report the synthesis of a ratiometric fluorescent amyloid sensor, AmyBlink-1 and demonstrate its utility in the nanoscale imaging of amyloid fibrils formed by different proteins. The design of AmyBlink-1 includes an amyloid reporter moiety and a photoswitching fluorophore (Fig. 1). ThT is widely applied for detection of diverse amyloids that present the typical cross- $\beta$ sheet architecture and was therefore chosen as the amyloid reporter moiety. ${ }^{[5,11,14]}$ The cyanine analogue Alexa Fluor 647 (AF647) was chosen as the photo-switching fluorophore for two reasons. Firstly, AF647 exhibits excellent photostability and photoswitching properties in buffer systems used for SMLM techniques. ${ }^{[15]}$ Secondly, its spectral separation from ThT allows for a ratiometric fluorescence response. ${ }^{[16]}$

AmyBlink-1 was synthesized by coupling ThT and AF647 via a cadaverine linker. The long linker was used to ensure the fluorescence properties of each fluorophore were unaffected by proximity effects (Fig. 1, Scheme S1).

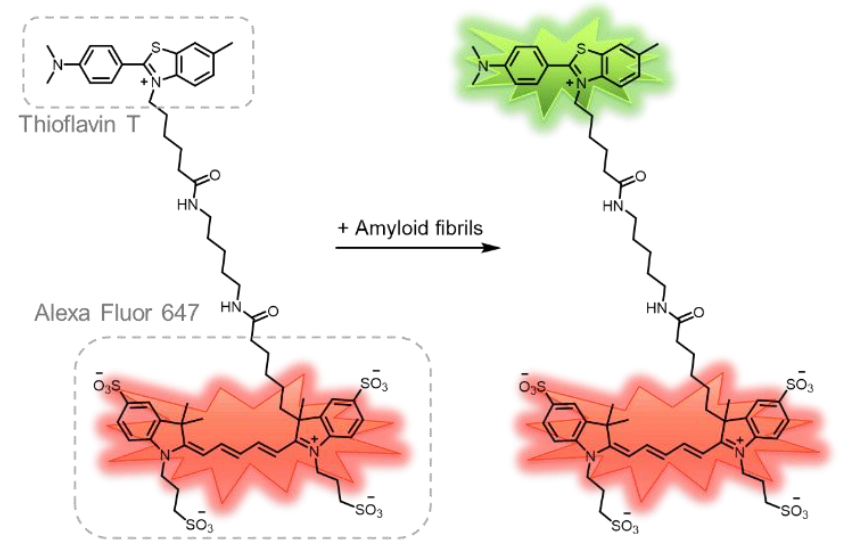

AmyBlink-1

Figure 1: Structure of AmyBlink-1, which comprises thioflavin T (ThT) tethered to AF647 via an aliphatic linker. The ThT group undergoes a fluorescence turnon upon binding to amyloid fibrils, while the AF647 emission is unchanged.

In the absence of protein, excitation of AmyBlink-1 at $440 \mathrm{~nm}$ resulted in weak fluorescence around $485 \mathrm{~nm}$ and strong fluorescence at $670 \mathrm{~nm}$ (Fig. 2(a)). We measured the absorbance and fluorescence properties of AmyBlink-1 in the presence of monomeric and fibrillar forms of amyloid-forming proteins. Insulin was chosen as an in vitro model amyloid system to study the sensing properties of AmyBlink-1 because insulin fibrils exhibit the characteristic cross- $\beta$ amyloid architecture and have been extensively studied ${ }^{[17]}$. Moreover, insulin fibril formation is responsible for injection-localized amyloidosis in patients prescribed with insulin therapy. ${ }^{[18]}$ We observed a 5-fold increase in the fluorescence intensity at $480 \mathrm{~nm}$ for AmyBlink-1 when incubated in the presence of fibrillar insulin compared to monomeric insulin and buffer (Fig. 2(a)). However, no significant change was detected in the fluorescence intensity at $670 \mathrm{~nm}$ (corresponding to AF647) when incubated with monomeric or fibrillar insulin compared to buffer (Fig. 2(a)). Similar trends were observed in the absorption profiles of AmyBlink-1 (Fig. S4). These observations are consistent with literature reports on the fluorescence of amyloid bound-Th ${ }^{[11]}$ and verify that tethering AF647 to ThT via an aliphatic linker does not alter its amyloidsensing properties. Upon excitation at $640 \mathrm{~nm}$, the emission profile of AmyBlink-1 remains unaltered in the presence of monomeric or fibrillar insulin when compared with the buffer control (Fig. S5). We then evaluated the ratiometric response of AmyBlink-1 towards monomeric and fibrillar species of amyloidbeta ( $A \beta_{40}$ and $A \beta_{42}$ isoforms) and tau protein $4 R$ isoform. The amyloid-beta peptide $(A \beta)$ is the main component of senile plaques in the brains of patients with $A D$, while tau proteins are predominant in the neurofibrillary tangles (NFTs) observed in tauopathies including PD. ${ }^{[19]}$ The ratio of ThT and AF647 intensities $\left(I_{480} / I_{670}\right)$ increased 5 to 6 -fold in the presence of amyloid fibrils (Fig. 2(b)), confirming AmyBlink-1 is responsive to diverse amyloid fibrils exhibiting the characteristic cross- $\beta$ architecture.

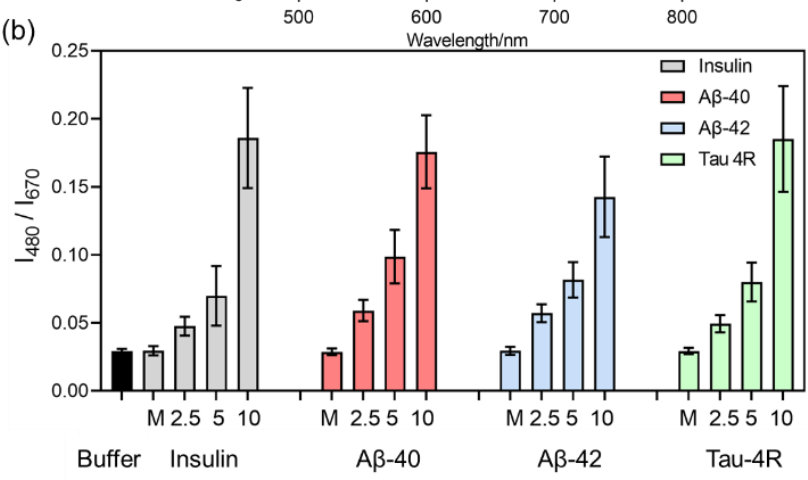

Figure 2: (a) Fluorescence spectra of AmyBlink-1 $(1 \mu \mathrm{M})$ in the presence of phosphate buffer ( $\mathrm{pH} 7.4)$, insulin monomer $(10 \mu \mathrm{M})$ and insulin fibrils $(10 \mu \mathrm{M})$, excitation at $440 \mathrm{~nm}$. Inset shows the emission profiles corresponding to thioflavin T. (b) Ratio of emission at 480 and $670 \mathrm{~nm}$ of AmyBlink-1 in the presence of phosphate buffer ( $\mathrm{pH}$ 7.4), monomeric insulin, $A \beta_{40}, A \beta_{42}$ and Tau proteins $(\mathrm{M})$ and their fibrils prepared from different concentration of proteins $(2.5,5$ and $10 \mu \mathrm{M})$

Elaboration of ThT with the AF647 moiety does not diminish its affinity for insulin and $A \beta_{40}$ fibrils. The binding affinities $\left(K_{d}\right)$ of AmyBlink-1 for insulin (Fig. S6) and $A \beta_{40}$ (Fig. S7) fibrils were determined to be $0.73 \pm 0.26 \mu \mathrm{M}$ and $0.98 \pm 0.36 \mu \mathrm{M}$ respectively. These values are very similar to those measured for unmodified ThT towards insulin $\left(0.76 \pm 0.21 \mu \mathrm{M}\right.$, Fig. S6) and $A \beta_{40}(0.92 \pm$ $0.28 \mu \mathrm{M}$, Fig. S7) and those reported in the literature. ${ }^{[20]}$ We next performed confocal microscopy which confirmed that the increase in fluorescence intensity results from binding of AmyBlink-1 to amyloid fibrils. Monomeric forms of insulin, $A \beta_{40}, A \beta_{42}$ and tau proteins incubated in the presence of AmyBlink-1 did not exhibit significant fluorescence (Fig. S8 - S11), while strong, colocalized fluorescence was observed in both green and red channels for insulin, $A \beta_{40}, A \beta_{42}$ and tau fibrils (Fig. S8 - S11).

To assess the suitability of AmyBlink-1 for super-resolution microscopy, we evaluated the performance of different buffer systems for dSTORM imaging, using insulin fibrils. ${ }^{[7]}$ We trialed a 
number of buffer additives: an oxygen scavenging system (glucose oxidase/catalase (GODCAT) or protocatechuic acid/protocatechuate-3,4-dioxygenase (PCA/PCD)); a reducing thiol ( $\beta$-mercaptoethanol (BME) or mercaptoethylamine (MEA)); and dye-stabilizing cyclooctatetraene (COT). ${ }^{[21]}$ We found that the PCA/PCD buffer resulted in the highest mean photon count for AmyBlink-1 in the presence of fibrils, $>3$-fold higher than GODCAT/MEA and 6-fold higher than GODCAT/BME (Fig. S12 and S13). Importantly, this came with no impairment of the number of localizations detected over 10,000 frames (Fig. S14). These results are consistent with reports that PCA/PCD buffers enhance dye performance in dSTORM imaging due to better $\mathrm{pH}$ stability during imaging and direct quenching of the triplet state by energy transfer. ${ }^{[22]}$ We subsequently used the PCA/PCD buffer for all dSTORM imaging with AmyBlink-1.

Next, we performed dSTORM imaging of insulin fibrils treated with AmyBlink-1. Images in Fig. 3 show a comparison of diffractionlimited fluorescence images of insulin fibrils (Fig. 3(a)) and the corresponding dSTORM images (Fig. 3(b)). Using AmyBlink-1, we achieved an 10 -fold improvement in resolution in the reconstructed dSTORM image compared to the diffraction limited image (Fig. 3 (c-e)), with calculated full width at half maximum (FWHM) values of $29 \mathrm{~nm}$ and $310 \mathrm{~nm}$ respectively (Fig. 3(e)). Amyloid fibrils typically are 8-12 nm wide, ${ }^{[23]}$ but the conjugation of AF647 to ThT through a flexible 2.5 nm linker in AmyBlink-1 impacts the resolution that can be achieved. The localization precision ${ }^{[24]}$ calculated from this data set was $8 \pm 2 \mathrm{~nm}$ in $\mathrm{x}$ and 9 $\pm 2 \mathrm{~nm}$ in y axes (Fig. S15).

Having verified that tethering AF647 to ThT via an aliphatic linker did not alter its photoswitching behavior and thereby its applicability for dSTORM imaging, we performed super-resolution imaging of insulin, $A \beta_{40}, A \beta_{42}$ and Tau fibrils treated with AmyBlink-1 and successfully obtained super-resolution images of the tested amyloids with measured fibril widths of $\sim 30-52 \mathrm{~nm}$, demonstrating the versatility of AmyBlink-1 as a probe for superresolution imaging of a variety of amyloid targets (Fig. S16).

Given the reported role of early amyloid assemblies in neurodegeneration, ${ }^{[3 b, 25]}$ we explored the utility of AmyBlink-1 to visualize intermediates formed at different stages of amyloid fibril formation. The assembly of fibrils was achieved in bulk solution (Fig. S17 and S20) in the absence of any sample-substrate interactions that might have affected the growth kinetics. ${ }^{[26]}$ Samples of insulin and $A \beta_{40}$ aggregates were removed at the end of the lag phase $\left(t_{1}\right)$, at two points in the elongation phase $\left(t_{2}\right.$ and $\left.t_{3}\right)$, and after the increase in ThT intensity plateaued $\left(t_{4}\right)$ and imaged in dSTORM mode with AmyBlink-1 (Fig. S17 and S22). Remarkably, dSTORM imaging using AmyBlink-1 enabled the fluorescent visualization of early-stage amyloid structures, at the point of elongation into fibrils (Fig. S18) and $A \beta_{40}$ (Fig. 4(a)), with dimensions of $50-300 \mathrm{~nm}$, through to mature fibrils with lengths greater than $\left.10,000 \mathrm{~nm} \cdot{ }^{[8 b}, 12 \mathrm{a}\right]$ To quantify heterogeneity in the nanoscale morphological features of insulin and $A \beta_{40}$ intermediates we performed cluster detection analyses of the dSTORM images using a hierarchical density-based algorithm, hierarchical density-based spatial clustering of applications with noise (HDBSCAN). From the cluster-maps generated for each dSTORM image of insulin (Fig. S18) and $A \beta_{40}$ (Fig. 4(b)) fibrils, we were able to extract quantitative information pertaining to the length, area, and skew (ratio of major to minor axis) of the clusters.
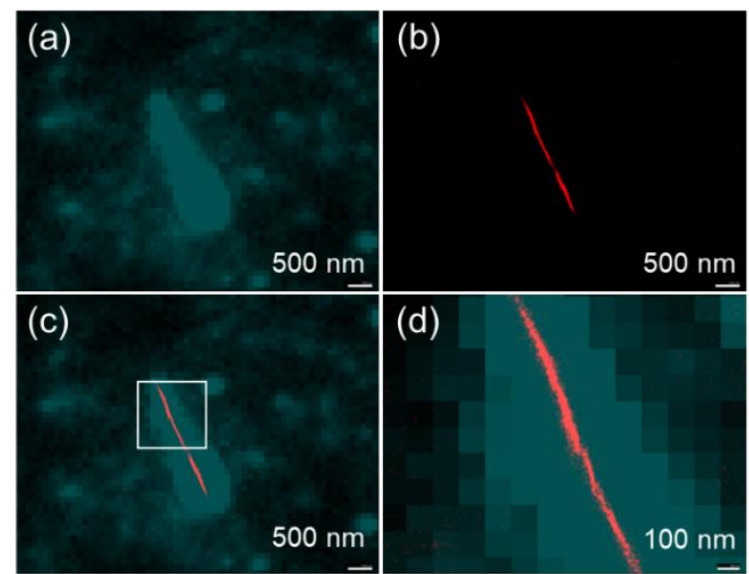

(e)

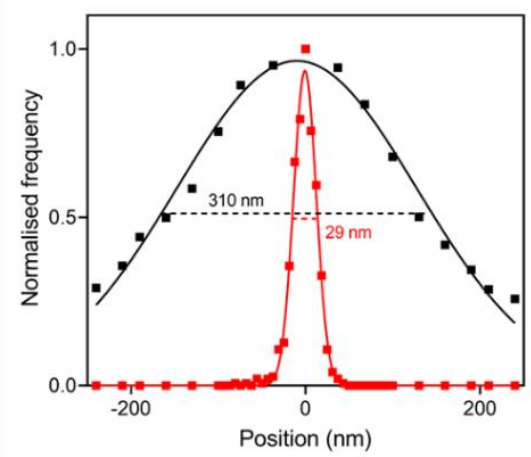

Figure 3: Comparison of conventional fluorescence and super-resolution images of insulin fibrils $(5 \mu \mathrm{M})$ with AmyBlink-1 (100 nM) (a) conventional (diffraction limited) fluorescence image of the fibrils (b) corresponding dSTORM image (c) overlay of the diffraction-limited and dSTORM image (d) image of the overlay denoted by white square demonstrating the 10-fold improvement in resolution compared to conventional imaging (e) cross-sectional profiles of conventional fluorescence (black) and dSTORM (red) super-resolution images of fibrils using AmyBlink-1 with the FWHM values of the distributions.

In Fig. 4(c), we report the distribution of the measured lengths of individual $A \beta_{40}$ amyloid aggregates at sampling points $t_{1}-t_{4}$ along the assembly pathway, detected from the cluster maps in dSTORM images. As expected, the width of the distribution profiles of the measured fibril lengths values increases with time for both insulin (Fig. S19) and $A \beta_{40}$ fibrils (Fig. 4(c)). The shift in the center of the distribution of fibril lengths reflects an increase in average fibril length along the assembly time-course, with an average fibril length of $600,1000,3900$ and $6100 \mathrm{~nm}$ at $t_{1}, t_{2}, t_{3}$ and $t_{4}$ respectively (Fig. 4(c)). Fig. S19 and S21 depict the histograms of the areas of individual aggregates at timepoints $t_{1}-$ $t_{4}$ for insulin and $A \beta_{40}$ aggregates. The distribution of the areas of aggregates calculated from the cluster maps demonstrate a similar trend with measured areas increasing across $t_{1}-t_{4}$ (Fig. S19 and S21). We further investigated the skew of individual amyloid aggregates (Fig. 4(d)), which represents the ratio of major to minor axis; with a value of 1 representing a sphere and those $>1$ corresponding to elongation along one of the axes (major axis). The mean skew values increased 1.5 -fold, from 1.5 to 2.3 , between the $t_{1}$ and $t_{2}$ phase, reflecting the conversion of a majority of spherical structures to those with a more distinct fibrillar morphology at the later time point. A striking shift in the skew distribution is observed between the profiles collected at $t_{2}$ and $t_{3}$ where the distribution changes from overwhelmingly zero-tailed to 
a more symmetrical shape (Fig. 4(d) and S19). This reflected in the calculated mean of the distributions at time-points $t_{2}$ and $t_{3}$, with the largest increase (3-fold) in mean skew values, from 2.3 to 7 between the $t_{2}$ and $t_{3}$ phases. Between the $t_{3}$ and plateau $t_{4}$ phases, the skew values of fibrils show a minimal increase (1.2fold) from a mean skew value of 7 to 8.5 , reflecting the shift from elongation to plateau phase of the amyloid assembly pathway. Other methods, including atomic force microscopy and electron microscopy, have revealed the transition from spherical to elongated morphology. Many studies have reported on oligomers with amorphous, prefibrillar, micellar or annular structures. ${ }^{[27]}$ Here, we demonstrate that AmyBlink-1 additionally allows visualization and quantification of the cross- $\beta$ structure at some of the earliest stages of amyloid assembly. The binding through the ThT moiety to structures with skew values close to 1 reveals that these oligomers have undergone a conformational conversion to a fibrillar structure that renders them competent for elongation into fibrils. ${ }^{[28]}$ These experiments demonstrate the ability of AmyBlink1 to probe the structure and morphological features of diverse amyloid proteins with nanometer resolution without the need for genetic modification or covalent-labelling, which are not only timeconsuming and labor-intensive, but have been reported to alter the assembly process of the amyloidogenic proteins. Moreover, sample preparation for dSTORM imaging with AmyBlink-1 is simple and direct, unlike that achieved with the negative stains often employed for transmission electron microscopy which often show variable efficiency of staining for different amyloids and where images can be distorted when large structures are densely stained. Using dSTORM imaging with AmyBlink-1, large sample areas can be imaged rapidly $(50 \times 80 \mu \mathrm{m})$, facilitating representative imaging that captures the full range of structures present within a heterogeneous sample.

(a)

(b)
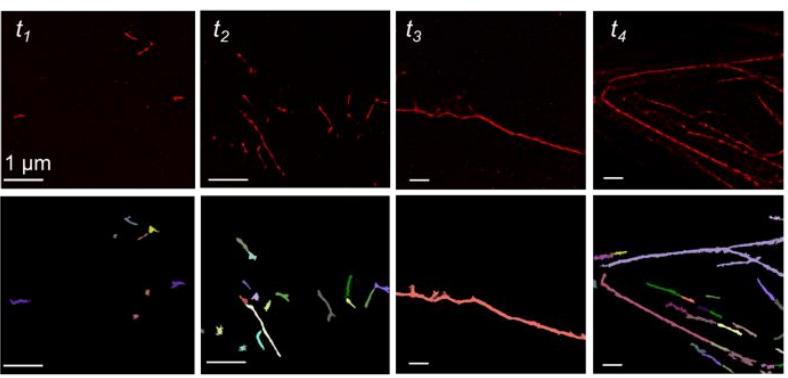

(c)
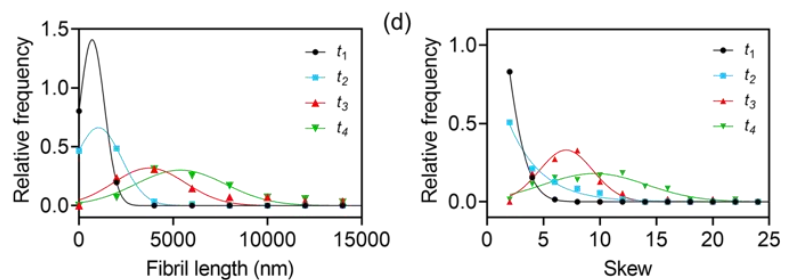

Figure 4: Super-resolution imaging and quantitative analysis of morphologies of $A \beta_{40}$ aggregates at different stages of aggregation. (a) dSTORM images of $A \beta_{40}$ aggregates imaged with AmyBlink-1 at late lag (t1), early-exponential (t2), late-exponential (t3) and plateau (t3) phases (b) corresponding cluster maps of dSTORM images from hierarchical density-based algorithm (HDBSCAN) (c) histograms of the distributions of lengths of individual aggregates at different time points. Bin size $=2000 \mathrm{~nm}$ (d) histograms of the skew (ratio of major and minor axis) of individual aggregates at different time points. Bin size $=2$.

Here we have presented AmyBlink-1, a fluorescent sensor designed for super-resolution imaging of amyloids. Photophysical and binding affinity studies demonstrate the desirable ratiometric sensing properties of AmyBlink-1 and versatility for detecting a range of amyloid-based structures. Incorporation of the photoswitching fluorophore, AF647, allows for application of AmyBlink-1 in super-resolution imaging experiments, facilitating imaging of amyloids with a resolution of $\sim 29 \mathrm{~nm}$. AmyBlink-1 therefore enables the imaging of some of the earliest structures committed to amyloid assembly. Quantitative analysis of the heterogeneity, on the nanoscale, during the formation and deposition of fibrils will open new doors to understanding the molecular mechanisms of amyloid-related pathologies.

\section{Acknowledgements}

The authors would like to acknowledge the Australian Research Council DE210101176 and the University of Sydney Fellowship (AK), CE200100012 and DP180101353 (EJN), and DP200102463 (MS) for funding. SRB was supported by a Research Training Program Scholarship from the Australian Government (Department of Education, Skills and Employment). We thank Dr Maximo Sanz-Hernandez for helpful discussions on cluster detection algorithms and Mr Max O.D.G. Baker for suggesting the name, AmyBlink-1 for the sensor. We acknowledge the scientific and technical assistance of staff from the Mass Spectrometry Facility at the School of Chemistry and Sydney Analytical and Sydney Microscopy and Microanalysis (SMM) Core Research Facilities at the University of Sydney.

Keywords: fluorescence $\cdot$ super-resolution $\bullet$ sensor $•$ amyloid $•$ oligomers

[1] (a) M. Sunde, L. C. Serpell, M. Bartlam, P. E. Fraser, M. B. Pepys, C. C. F. Blake, Journal of Molecular Biology 1997, 273, 729-739; (b) M. R. Sawaya, M. P. Hughes, J. A. Rodriguez, R. Riek, D. S. Eisenberg, Cell 2021, 184, 4857-4873.

[2] F. Chiti, C. M. Dobson, Annual Review of Biochemistry 2017, 86, 27-68.

[3] (a) F. Bemporad, F. Chiti, Chemistry \& Biology 2012, 19 315-327; (b) M. E. Larson, S. E. Lesné, Journal of Neurochemistry 2012, 120, 125-139.

[4] (a) I. Benilova, E. Karran, B. De Strooper, Nature Neuroscience 2012, 15, 349-357; (b) M. T. FoderoTavoletti, V. L. Villemagne, C. C. Rowe, C. L. Masters, K. J. Barnham, R. Cappai, The International Journal of Biochemistry \& Cell Biology 2011, 43, 1247-1251.

[5] A. Kaur, E. J. New, M. Sunde, ACS Sensors 2020, 5 2268-2282.

D. Baddeley, J. Bewersdorf, Annual Review of Biochemistry 2018, 87, 965-989.

[7] M. J. Rust, M. Bates, X. Zhuang, Nature methods 2006, 3 793-795.

[8] (a) F. Cassé, S. Martin, Vol. 9, 2015, pp. 367-367; (b) D. Pinotsi, A. K. Buell, C. Galvagnion, C. M. Dobson, G. S. Kaminski Schierle, C. F. Kaminski, Nano Letters 2014, 14 339-345.

[9] S. J. Sahl, S. W. Hell, S. Jakobs, Nature Reviews Molecular Cell Biology 2017, 18, 685-701.

[10] (a) A. Kaur, M. A. Haghighatbin, C. F. Hogan, E. J. New, Chemical Communications 2015, 51, 10510-10513; (b) A. Kaur, K. Jankowska, C. Pilgrim, S. T. Fraser, E. J. New, Antioxidants and Redox Signaling 2016, 24, 667-679. 
[11] M. Biancalana, S. Koide, Biochimica et biophysica acta 2010, 1804, 1405-1412.

[12] (a) K. Spehar, T. Ding, Y. Sun, N. Kedia, J. Lu, G. R. Nahass, M. D. Lew, J. Bieschke, ChemBioChem 2018, 19, 1944-1948; (b) H. Huh, J. Lee, H. J. Kim, S. Hohng, S. K. Kim, Chemical Physics Letters 2017, 690, 62-67; (c) J.

Ries, V. Udayar, A. Soragni, S. Hornemann, K. P. R. Nilsson, R. Riek, C. Hock, H. Ewers, A. A. Aguzzi, L. Rajendran, ACS Chemical Neuroscience 2013, 4, 10571061; (d) J.-E. Lee, J. C. Sang, M. Rodrigues, A. R. Carr M. H. Horrocks, S. De, M. N. Bongiovanni, P. Flagmeier, C. M. Dobson, D. J. Wales, S. F. Lee, D. Klenerman, Nano Letters 2018, 18, 7494-7501.

[13] (a) M.-C. Cui, M. Ono, H. Watanabe, H. Kimura, B.-L. Liu, H. Saji, Journal of the American Chemical Society 2014, 136; (b) T. J. Eckroat, A. S. Mayhoub, S. GarneauTsodikova, Beilstein Journal of Organic Chemistry 2013, 9, 1012-1044.

[14] C. Gan, L. Zhou, Z. Zhao, H. Wang, Medicinal Chemistry Research 2012, 22.

[15] (a) F. M. Jradi, L. D. Lavis, ACS Chemical Biology 2019 14, 1077-1090; (b) M. Minoshima, K. Kikuchi, Journal of Biological Inorganic Chemistry 2017, 22, 639-652.

[16] (a) C. Shen, J. L. Kolanowski, C. M. N. Tran, A. Kaur, M. C. Akerfeldt, M. S. Rahme, T. W. Hambley, E. J. New, Metallomics 2016, 8, 915-919; (b) A. Kaur, J. L.

Kolanowski, E. J. New, Angewandte Chemie International Edition 2016, 55, 1602-1613.

[17] D.-P. Hong, A. Ahmad, A. L. Fink, Biochemistry 2006, 45, 9342-9353.

[18] S. Okamura, Y. Hayashino, S. Kore-Eda, S. Tsujii, Diabetes Care 2013, 36, e200.

[19] M. G. ladanza, M. P. Jackson, E. W. Hewitt, N. A. Ranson, S. E. Radford, Nature Reviews Molecular Cell Biology 2018, 19, 755-773.

[20] (a) F. Peccati, S. Pantaleone, V. Riffet, X. Solans-Monfort, J. Contreras-García, V. Guallar, M. Sodupe, The Journal of Physical Chemistry B 2017, 121, 8926-8934; (b) W. E. Klunk, Y. Wang, G.-f. Huang, M. Debnath, D. Holt, C. Mathis, Life Sciences 2001, 69, 1471-1484.

[21] G. T. Dempsey, J. C. Vaughan, K. H. Chen, M. Bates, X. Zhuang, Nature Methods 2011, 8, 1027-1040.

[22] N. Olivier, D. Keller, P. Gönczy, S. Manley, PLOS ONE 2013, 8, e69004.

[23] F. Chiti, C. M. Dobson, Nature Chemical Biology 2008, 5, 15-15.

[24] B. Rieger, S. Stallinga, ChemPhysChem 2014, 15, 664670.

[25] C. G. Glabe, R. Kayed, Neurology 2006, 66, S74 LP-S78.

[26] (a) J. Legleiter, in Alzheimer's Disease and Frontotemporal Dementia: Methods and Protocols (Ed.: E. D. Roberson), Humana Press, Totowa, NJ, 2011, pp. 5770; (b) W. Hoyer, D. Cherny, V. Subramaniam, T. Jovin, Biochemistry 2005, 43, 16233-16242.

[27] C. G. Glabe, Journal of Biological Chemistry 2008, 283, 29639-29643.

[28] T. C. T. Michaels, A. Šarić, S. Curk, K. Bernfur, P. Arosio, G. Meisl, A. J. Dear, S. I. A. Cohen, C. M. Dobson, M. Vendruscolo, S. Linse, T. P. J. Knowles, Nature Chemistry 2020, 12, 445-451. 
